\title{
MOZART EFFECT AND MUSIC PSYCHOLOGY: RECENT DEVELOPMENTS AND FUTURE RESEARCH
}

\author{
Vaitsa Giannouli \\ Aristotle University of Thessaloniki, Greece \\ E-mail: giannouliv@hotmail.com
}

The field of Music Psychology has grown in the past 20 years, to emerge from being just a minor topic to one of mainstream interest within the brain sciences (Hallam, Cross, $\&$ Thaut, 2011). Despite the plethora of research attempts to examine the so-called hotly disputed "Mozart effect" which was first reported by Rauscher, Shaw, and Ky $(1993,1995)$, we still know little about it. This group of researchers were the first to support experimentally that visuospatial processing was enhanced in participants following exposure to Mozart's Sonata for Two Pianos in D major (K.448). Although the first research attempts referred to the Mozart effect as an easy way of improving cognitive performance immediately after passive music listening to Mozart's sonata K. 448 (Chabris, 1999), after which healthy young adult students could perform with enhanced spatialtemporal abilities in tasks such as the Paper Folding Task (PFT), nowadays there is a number of studies indicating that this specific music excerpt does not necessarily have this magical influence on all cognitive abilities (e.g. on the overall Intelligence Quotient) in humans and on the behavior of animals (for a review see Giannouli, Tsolaki \& Kargopoulos, 2010). In addition to that, questions arise whether listening to this 'magic music excerpt' does indeed have benefits that generalize across a wide range of cognitive performance, and if it can induce changes that are of importance for medical and therapeutic purposes in patients with neurological disorders (e.g. epilepsy) or psychiatric disorders (e.g. dementia, depression) (Verrusio et al., 2015).

Although two meta-analyses in the past support the existence of a moderate influence on cognitive tasks after brief listening to Mozart's ten-minute sonata (Chabris, 1999; Hetland, 2000), more studies are needed, in order to define specific protocols that result in these claimed changes (Verrusio et al., 2015). For example, there are recent studies claiming that Mozart's K. 448 sonata can improve mood (Giannouli, 2013; Giannouli, Lytras \& Syrmos, 2012), but not verbal memory (Giannouli, Tsolaki \& Kargopoulos, 2010; Giannouli, Kolev \& Yordanova, 2018), visuospatial short-term memory (Giannouli \& Popa, 2017), and attention (Giannouli, 2012) across young and old healthy adults.

A possible explanation for the (in)existence of the Mozart effect may be found in the fact that music elicits a variety of emotions and the way music excerpts are emotionally perceived by each individual. Thus, Mozart effect may be associated with any form of music that has or is perceived as having energetic and positive emotional qualities for the participants, and not necessarily specific structural-morphological characteristics (Thompson, Schellenberg \& Husain, 2001).

Given the heterogeneity of the relevant research studies and the contrasting results, future research should further focus in a more systematic way not only in replicating the methods employed in previous studies (e.g. if music is preceding or used as background, the types-genres of music excerpts that are used for comparing the different experimental conditions), but also on clarifying other aspects of this claimed phenomenon such as the individual characteristics that listeners have, and more specifically the role of music-induced emotions (Thompson, Schellenberg \& Husain, 2001), the role of music attitudes (Giannouli, 2018), as well as other demographic factors that differentiate individuals' cognitive and emotional reactions, such as age, gender, health status and music education that participants have received in their life (Giannouli, Kolev \& Yordanova, 2018). But the unanswered question still remains... Is there actually a 'Mozart effect'? 


\section{References}

Campbell, D. (2009). The Mozart effect: Tapping the power of music to heal the body, strengthen the mind, and unlock the creative spirit. New York: Harper Collins.

Chabris, C. (1999). A quantitative meta-analysis of Mozart studies. Nature, 400, 826-27.

Giannouli, V. (2012). Attention and Music. In E. Cambropoulos, C. Tsougras, P. Mavromatis \& K. Pastiadis (Eds.), Paper presented at the Proceedings of the $12^{\text {th }}$ International Conference on Music Perception and Cognition and the $8^{\text {th }}$ Triennial Conference of the European Society for the Cognitive Sciences of Music (pp. 345), Thessaloniki: Aristotle University of Thessaloniki.

Giannouli, V. (2013). Attitudes towards music as a means of therapy: Can it help to overcome depression and/ or cardiovascular disease? Health Psychology Research, 1 (1). Retrieved from https://www.ncbi.nlm. nih.gov/pmc/articles/PMC4768609.

Giannouli, V. (2018). Exploring passive music listening and its impact on cognition: Attitudes in focus. Journal of Cognitive Neuropsychology, 1 (4), 1.

Giannouli, V., Kolev, V., \& Yordanova, J. (2018). Is there a specific Vivaldi effect on verbal memory functions? Evidence from listening to music in young and old adults. Psychology of Music, (accepted in press).

Giannouli, V., Lytras, N., \& Syrmos, N. (2012). Is there a place for music in nuclear medicine. Hellenic Journal of Nuclear Medicine, 15 (3), 188-189.

Giannouli, V., \& Popa, S. (2017). Does listening to Mozart's music influence visuospatial short-term memory in young adults? European Psychiatry, 41, S684.

Giannouli, V., Tsolaki, M., \& Kargopoulos, P. (2010). The influence of Mozart's and Beethoven's music on reverse mnemonic recall tasks. Psychiatrike, 21 (1), 60-67.

Hallam, S., Cross, I., \& Thaut, M. (Eds.). (2011). Oxford handbook of music psychology. Oxford: Oxford University Press.

Hetland, L. (2000). Listening to music enhances spatial-temporal reasoning: Evidence for the" Mozart Effect". Journal of Aesthetic Education, 34 (3/4), 105-148.

Rauscher, F. H., Shaw, G. L., \& Ky, C. N. (1993). Music and spatial task performance. Nature, 365 (6447), 611-611.

Rauscher, F. H., Shaw, G. L., \& Ky, K. N. (1995). Listening to Mozart enhances spatial-temporal reasoning: Towards a neurophysiological basis. Neuroscience Letters, 185 (1), 44-47.

Thompson, W. F., Schellenberg, E. G., \& Husain, G. (2001). Arousal, mood, and the Mozart effect. Psychological Science, 12 (3), 248-251.

Verrusio, W., Moscucci, F., Cacciafesta, M., \& Gueli, N. (2015). Mozart Effect and its clinical applications: A review. British Journal of Medicine and Medical Research, 8 (8), 639-650.

Received: November 12, 2017

Accepted: December 18, 2017

Vaitsa Giannouli $\quad \mathrm{PhD}$, Researcher, School of Medicine, Aristotle University of Thessaloniki,

Thessaloniki, Greece.

Email: giannouliv@hotmail.com 